\title{
Construction of Control Nodes in Wireless Sensor Network based on Improved Model Predictive Control Algorithm
}

\author{
Qing $\operatorname{Tan}^{1, a}$ \\ ${ }^{1}$ College of Information Technology, Luoyang Normal University, Luoyang, 471934, China \\ aedutanqing@163.com
}

\begin{abstract}
Keywords: Model predictive control algorithm; Wireless sensor network; Sensor node; Control Nodes; Topology
\end{abstract}

\begin{abstract}
Model predictive control algorithm is of three characteristics: predictive model, receding horizon optimization, feedback correction. The sensor node consists of processing module, communication module, sensor module and power supply module. This paper analyzes and improves model predictive control algorithm. Development process control is the development and control theory, instrumentation, computer communication and network. The paper proposes construction of control nodes in wireless sensor network based on improved model predictive control algorithm. Finally, the effectiveness of the proposed method is proved by experiments.
\end{abstract}

\section{Introduction}

At present, in the theory and the control algorithm has been studied deeply and widely. Although in the university analysis has made some achievements, but in the multi variable algorithm, research on the stability and robustness is relatively deficient, can only be studied by simulation and the tentative application. According to the characteristics of nonlinear industrial process there is a lot of time varying system, nonlinear predictive control and robust predictive control is still the focus of future research.

With low energy consumption and the expanding network routing and routing scalability, strong adaptability to dynamic network structure, routing protocol is more suitable for the characteristics of wireless sensor networks [1]. In the design of network layer routing problems: whether the path to the traditional network that the selection of the optimum routing performance indicators, to measure the consistency as compared to traditional network.

Research and development of predictive control framework, has been a breakthrough in early research, get rid of the monotone algorithm model, which began with the pole assignment, adaptive control, robust control, linearization, decoupling control and nonlinear control of a class of advanced combination of predictive control strategy research; and with the development of technology of intelligent control, prediction control will also be intelligent predictive control direction, such as fuzzy predictive control, neural network predictive control, genetic algorithms of predictive control, predictive control and self learning.

The data link layer protocol used to establish reliable point-to-point or point to multipoint communication link, including media access control (MAC) and error control [2]. The current mainstream wireless network technology, including mobile cellular network, Ad hoc and Bluetooth technology, media access control used (MAC protocol) is not suitable for wireless sensor networks. Among them, the cellular network is concerned with how to satisfy the requirement of quality of service and save the bandwidth resource; Ad hoc network to solve the connection establishment of a certain quality of service guarantee is highly mobile nodes in the environment, power is not a question of the first importance. But as the predictive model based, only a rough description of dynamic characteristics of the objects, because of the nonlinear, time variable exists in practical systems, the model mismatch, interference and other factors, based on the time invariant forecasting output model may not be fully consistent with the actual situation, it needs to supplement the shortage of forecast models for forecasting means additional, or based on the model is modified online. The paper proposes 
construction of control nodes in wireless sensor network based on improved model predictive control algorithm.

\section{Wireless Sensor Network for Control Nodes}

Control and a feature is the online optimization model. Predictive control optimization control algorithm is to determine the optimal future control effect through performance indicators of this. The performance index system related to future behavior, for example, it is usually desirable object tracking a desired trajectory in the sampling point in the future on the minimum variance, but also desirable wider form, such as requiring a minimum energy control while maintaining output at a given range. The future behavior of the system relates to performance indicators, according to the prediction model are decided by the control of the future.

Nodes are divided into ordinary sensor nodes to the cluster head node and data fusion, sensor node data to send to the cluster head node, and then by the cluster head nodes converge to the background. The cluster head nodes need to do more work, more energy consumption. If you use the same node cluster, according to the need to replace the cluster head, avoid the cluster head node death because of the transition energy consumption and it [3]. Add a layer of fixed wireless network Mesh mode formed in the network of sensor nodes, sensor nodes to collect data, on the other hand, realize the information communication between nodes, and the network fusion. Akyildiz L F summed up the application model of wireless Mesh network.

Wireless sensor network node according to the ability to move can be divided into static and mobile networks. In a static network: the network, nodes do not have the ability to move, when placed in the position cannot be changed [4]. Topology changes is small, usually because of energy depletion or damage caused by the node failure, or interference environment, terrain, obstacles to unreachable node, or a new node joins will lead to the change of network topology, power control and hierarchical clustering are often used as this kind of network topology control method. In the mobile network, the node with the ability to move can pass the path to reach the target position.

$$
E\left(k_{1}\right)=E_{\text {hui }}, E\left(k_{2}\right)=E_{\text {tidu }}
$$

In the flat topology, all nodes are equal, therefore, to plane structure called peer to peer structure. The planar structure has the characteristics of strong robustness, survivability, but with the increase of the size of the network, the network performance will decline rapidly. When the network size increases to a certain extent, the routing protocol may consume all of the network bandwidth, that is increasing the number of nodes in wireless sensor networks, network management and control overhead will gradually increase, thereby greatly reducing the overall performance of the network. The formula used in the method is as follows.

$$
y(t)=\sum_{j=1}^{n} c^{y}{ }_{j}(t)+y_{n}(t)
$$

Research on Clustering Algorithm in wireless sensor networks, most of them are studied in a homogeneous model of network clusters, such as how to make the number of nodes in the cluster, the cluster size as the same as the same, on this basis, effectively reduce the energy consumption. Most of the clustering algorithm using multi hop data will be transmitted to the base station, the base station distance nodes closer not only to collect the transport node in this cluster the data, but also to forward other than data from the cluster head nodes transmitting station far, this makes the distance closer to the base station node consume more energy than the cluster head nodes far away from the base station.

Sensor nodes in the process of data transmission in wireless channel competition, sharing, may cause the transmission between multiple nodes data collision, failed to send data packets, the praise of the sending and receiving data on the energy wave, and then need to repeat the transmitted data, thus more energy consumption of nodes. Crosstalk (Overhearing) data packet is transmitted in the wireless 
channel sharing, a node may receive and process not send their packets, at this time, a wireless receiving module and processor module will cause the node consumes more energy [5].

In wireless sensor networks, due to the RF chip communication distance constraints, a single node cannot know all the nodes in the network, can only know some information of its neighbor nodes. Therefore the first step to build a routing tree, let node understanding level and the parent node of the. SINK first by flooding to the network communication routing tree construction message. A message consists of a flag, a transmitting node ID, and the sending node level. Received the message send node a node ID records the parent node, and then the general value plus 1, and update the news spread to neighboring nodes to the ID and level, until all the nodes in the network have their parent node and level.

$$
X^{\prime}=W(X, P)=\left(\begin{array}{ccc}
a_{1} & a_{2} & d_{1} \\
a_{3} & a_{4} & d_{2} \\
0 & 0 & 1
\end{array}\right)\left(\begin{array}{l}
x \\
y \\
1
\end{array}\right)
$$

The sensor node consists of processing module, communication module, sensor module and power supply module, of which the first three modules is the main energy consumption module. The following according to the module introduces are several main energy saving strategy. Very low because the sensor module can play, so this is not discussed [6]. The processing module comprises a microprocessor and memory, the energy consumption can be lowered processing module idle condition through dynamic power management and dynamic voltage regulator two strategies, energy saving. Communication module mainly refers to the wireless transceiver, close communication module design and network protocol, the communication module in saving energy, but also analysis of sensor network protocols from the angle of energy management.

Sensor nodes are usually is a miniature of the embedded system, constitute the basic layer of wireless sensor network platform, looked from the network function, each sensor node is both traditional network node of the terminal and router functions, in addition to local information collection and the data processing, but also for the storage, management and processing integration to other nodes the data to complete some specific tasks, and collaborating with other nodes.

The network for each sensor node in the same fixed communication distance of $\mathrm{R}$ with its neighbor nodes communication; communication distance of $\mathrm{R}$ affects the positioning accuracy of sensor nodes, only $\mathrm{r}$ of moderate size can achieve better positioning accuracy; positioning accuracy in monitoring area depends on network connectivity and node beacons [7]. For the sensor network environment, the beacon node artificial deployed a large number of known location is not practical, but in the network layout of a certain proportion of GPS with the beacon nodes will increase the cost of positioning network is defined as:

$$
\begin{aligned}
& X(k \mid k-1)=A X(k-1 \mid k-1)+B u_{k-1} \\
& P(k \mid k-1)=A P(k-1 \mid k-1) A^{T}+Q
\end{aligned}
$$

Wireless sensor network consists of sensor nodes self organization, sensor nodes may exist the problem of heterogeneous, but from a mathematical graph theory and the research point of view, without considering the spatial difference situations can be the node of wireless sensor network is abstracted as the vertices of a graph, planar graph as follows is the plane of a wireless sensor network.

\section{Design structure of Model Predictive Control System}

Predictive control is a control algorithm based on the model; this model is called the prediction model. For the predictive control, only pay attention to the function of the model, and not to model, prediction model according to the object function is the historical information and future forecast on the future output input [8]. From a methodological perspective, as long as it is set with the prediction function of the information, regardless of what kind of form, can be used as the prediction model. 
Application of advanced process control technology from the current situation, in the next few years, predictive control, fuzzy control, control, intelligent control, adaptive control, soft sensor will become the mainstream of the development of expert system for advanced control software, will China's chemical industry, metallurgy, papermaking, pharmaceutical, petrochemical and other industries technological transformation, technological progress plays a positive role in promoting [9]. Development process control is the development and control theory, instrumentation, computer, computer communication and network and other disciplines closely related, as is shown by equation5.

$$
\phi(t)=\sqrt{2} \sum_{i} h(k) \phi(2 t-i)
$$

Prediction of infinite horizon LQR algorithm makes the algorithm with stable characteristics. For arbitrary linear objects, as long as the weighting matrices $\mathrm{Q}$ and $\mathrm{R}$ is positive definite, then the algorithm is proved to be stable (stationary and detection). State the object through the duality theory of estimation from noisy input and output measurements, now known as Kalman filter. The LQR controller and the Kalman filter combination is known as linear Gauss two time controller (LQG). In the LQG theory does not consider the input, output and the state constraints.

\section{Experiments and Analysis}

The prediction of optimal control and the traditional control are very different, it uses the concept of heuristic optimization, allows the designer the freedom to choose the form of the performance index. Therefore, for the same object, if the performance index by time domain parameters, different weighting matrix, it may cause the control effect is completely different. On the one hand, for the design of control system increases the degree of freedom, but the lack of design experience of people do not know where to start.

DMC using the step response model, while the MAC uses impulse response model; DMC control and MAC control increment. If the weighting is to the control action, then MAC has static difference. This problem can be solved by a static compensator. If you are not weighted, in the treatment of non minimum phase system must use a comprehensive method [10]. No control time domain $\mathrm{M}$ this one parameter MAC, namely control time $\mathrm{M}$ is equal to the prediction horizon estimation $\mathrm{P}$; DMC interference (2) is filtered, is a measure of value is the predicted value.

The wireless sensor network consists of several nodes, 1 node, 1 relay, 1 PC control center, system structure. Acquisition of wireless sensor network node to complete data acquisition, processing and communication work; the initiation and maintenance of sink node is responsible for the network, collect and upload data, will relay commands down sent notice acquisition node; transfer device sends the collected data and transfer the control center sends a command to the sink node; the control center is responsible for the final upload data, and users can give network operations command, as is shown by Fig. 1.

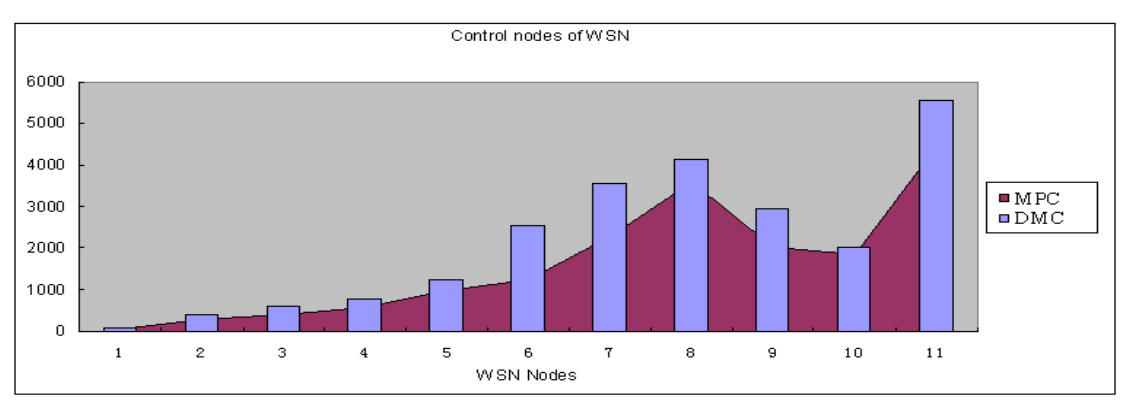

Figure 1. Comparison model predictive control algorithm in control nodes of wireless sensor network with DMC 
DMC algorithm is a kind of predictive control algorithm of object based on step response, which is suitable for the asymptotic stability of linear objects. For weakly nonlinear object, can be at work point first linearization; for unstable object can use state space model based on step response.

\section{Summary}

The paper proposes construction of control nodes in wireless sensor network based on improved model predictive control algorithm. Stability in the sense of MPC requirements than it is defined in the linear control theory to be more. First of all, because we are in theory to solve an infinite horizon control rate, type stability we seek is asymptotically stable error here; target and control variables will advance with time, infinitely small.

\section{References}

[1] Xiaoning Du, Yugeng Xi, Shaoyuan Li. An Efficient Constrained Model Predictive Control Algorithm Based on Approximate Computation. Journal of Systems Engineering and Electronics. 2002,13(1): 42-47

[2] Shermin S., Kashwan K. R., "An EFSM Based Fault Detections for Wireless Sensor Networks", JCIT, Vol. 10, No. 6, pp. $61 \sim 69,2015$.

[3] Guorui Li, Wenbo Shi, Ying Wang, Automatic ARIMA Time Series Modeling for Data Aggregation in Wireless Sensor Networks, JDCTA, Vol. 6, No. 23, pp. $438 \sim 447,2012$.

[4] Zeyad Ghaleb Al-Mekhlafi, Zurina Mohd Hanapi, Mohamed Othman, and Zuriati Ahmad, "Research and development on aspects of clock synchronization in a wireless sensor network", IJACT, Vol. 5, No. 14, pp. 62 76, 2013.

[5] Te-Jen Su, Tung-Yeh Tsou, Hong-Quan Vu, Wen-Jye Shyr, "Model Predictive Control Design Based on Particle Swarm Optimization", JCIT, Vol. 10, No. 6, pp. $70 \sim$ 83, 2015.

[6] D.Q. Mayne, J.B. Rawlings and C.V. Rao Constrained Model Predictive Control: Stability and Optimality. Automatic. 2000, 36(6) $789-814$.

[7] Li Shi-hui, "Research on The Wireless Sensor Network Mobile Node Localization Optimization Technology", JDCTA, Vol. 7, No. 4, pp. $305 \sim$ 312, 2013.

[8] L. Magni, G. de Nicolao, L. Magnani, et al. A Sabilizing Model- based Predictive Control Algorithm for Nonlinear Systems. Automatic. 2001, 37(9): 1351 -1362.

[9] Zhang Xizheng, Tang Yongqi, Wang Yinxu, "Model Predictive Control Allocation Based Coordinated Vehicle Dynamics Control with In-Wheel Motors", IJACT, Vol. 4, No. 3, pp. 75 83, 2012.

[10] Jiawei Huang, Xiangli Zhang, , Jin Ye, Local and Globe Cross-layer Congestion Control in Wireless Sensor Network, IJACT, Vol. 4, No. 11, pp. 311 317, 2012. 